AIAA 2000-1695

\title{
FIN BUFFETING FEATURES OF AN EARLY F-22 MODEL
}

\author{
Robert W. Moses, AIAA Member \\ Aeroelasticity Branch \\ NASA Langley Research Center \\ Hampton, VA
}

\begin{abstract}
Fin buffeting is an aeroelastic phenomenon encountered by high performance aircraft, especially those with twin vertical tails that must operate at high angles of attack. This buffeting is a concern from fatigue and inspection points of view. To date, the buffet (unsteady pressures) and buffeting (structural response) characteristics of the F-15 and F/A-18 fins have been studied extensively using flow visualization, flow velocity measurements, pressure transducers, and response gages. By means of windtunnel and flight tests of the F-15 and F/A-18, this phenomenon is well studied to the point that buffet loads can be estimated and fatigue life can be increased by structural enhancements to these airframes. However, prior to the present research, data was not available outside the F-22 program regarding fin buffeting on the F-22 configuration. During a test in the Langley Transonic Dynamics Tunnel, flow visualization and unsteady fin surface pressures were recorded for a $13.3 \%$-scale F-22 model at high angles of attack for the purpose of comparing with results available for similar aircraft configurations. Details of this test and fin buffeting are presented herein.
\end{abstract}

\section{$\underline{\text { Introduction }}$}

For high performance aircraft, such as the F/A-18, at high angles of attack, vortices emanating from wing leading edge extensions (LEX) often burst, immersing the vertical tails in their wake (Figure 1). Although these vortices increase lift, the resulting buffet loads on the vertical tails are a concern from airframe fatigue and maintenance points of view.

Copyright $\odot 2000$ by the American Institute of Aeronautics and Astronautics, Inc. No copyright is asserted in the United States under Title 17, U. S. Code. The U. S. Government has a royalty-free license to exercise all rights under the copyright claimed herein for Governmental Purposes. All other rights are reserved by the copyright owner.
Lawrence Huttsell, AIAA Associate Fellow

Design \& Analysis Branch

Air Force Research Laboratory

Wright-Patterson Air Force Base, OH

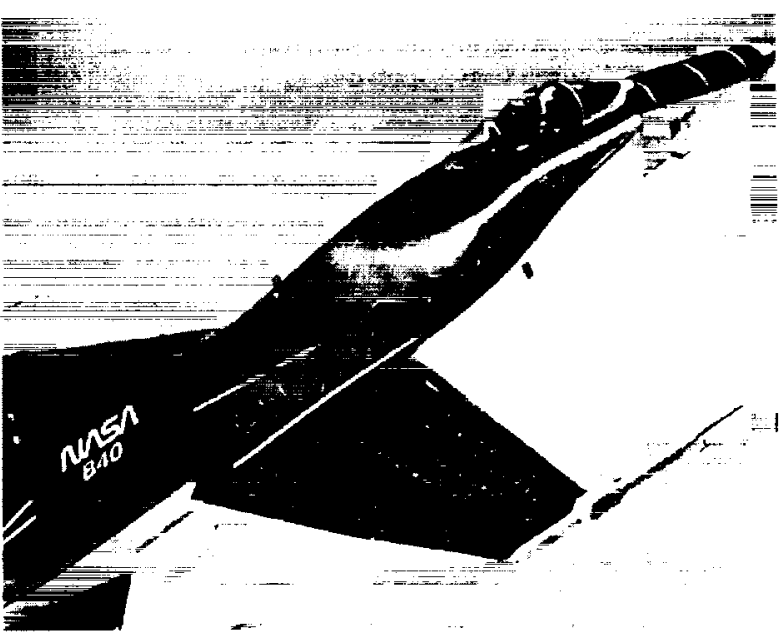

Figure 1. Flow Visualization of Vortex from the LEX Bursting ahead of the Vertical Tail on an F/A-18

(Photograph Courtesy of the NASA Dryden Flight Research Center)

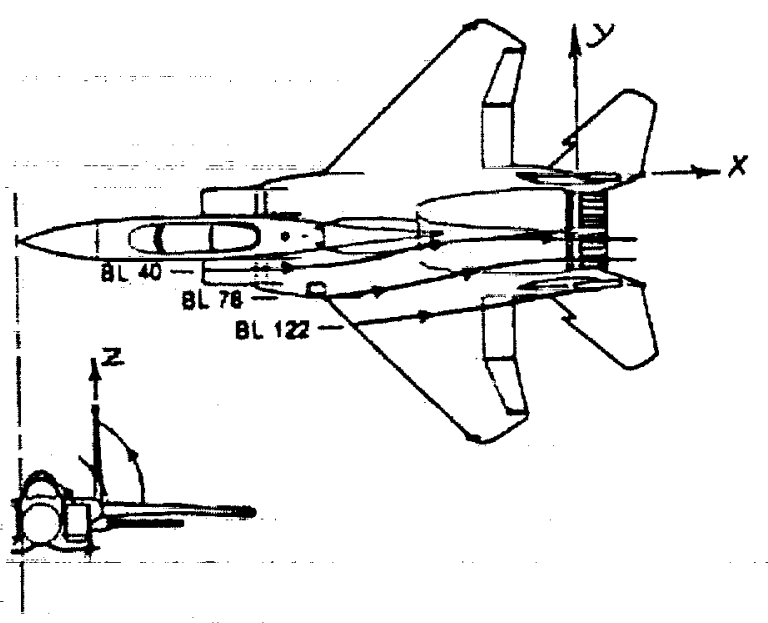

Figure 2. F-15 Eagle at 22 Degrees Angle of Attack, Showing Typical Trajectories of the Flow Affecting the Vertical Stabilizers (from Triplet et.al.)

As shown in Figure 1 for the F/A-18, the source of the buffet stems solely from one dominant LEX vortex that bursts ahead of the vertical tails. However, for the F-15 configuration (Figure 2), the buffet is created by the 
combination of several vortices originating from different sources which include the engine inlet and the wing leading edge as indicated by the trajectories. Because the configurations of the two vehicles differ, worst-case buffeting occurs in different modes. The F-15 fin suffers from high responses in its first torsion mode whereas the F/A-18 fin suffers from high responses in its first bending mode. Thus, configuration of the aircraft plays a vital role in the buffet and buffeting characteristics of the fin.

Previous wind-tunnel and flight tests ${ }^{1-10}$ have been conducted to quantify the buffet loads on the vertical tails of the F-15 and F/A-18. These tests were designed to characterize the flow mechanism and to quantify the unsteady differential pressures acting on the vertical tails during high-angle-of-attack maneuvers. The major findings of these tests were: I) that the buffet pressures vary with flight conditions; 2 ) that the buffeting (response of the tail) varies with flight conditions; and 3) that the power spectra scale with Strouhal number. Specifically for the F/A-18, later comparisons among pressure data from reducedscale wind-tunnel, full-scale wind-tunnel, and flight tests revealed that the time delays also scale with Strouhal number ${ }^{8-10}$. Using this new information, it was possible to estimate more accurately the fin buffeting for the F/A-18.

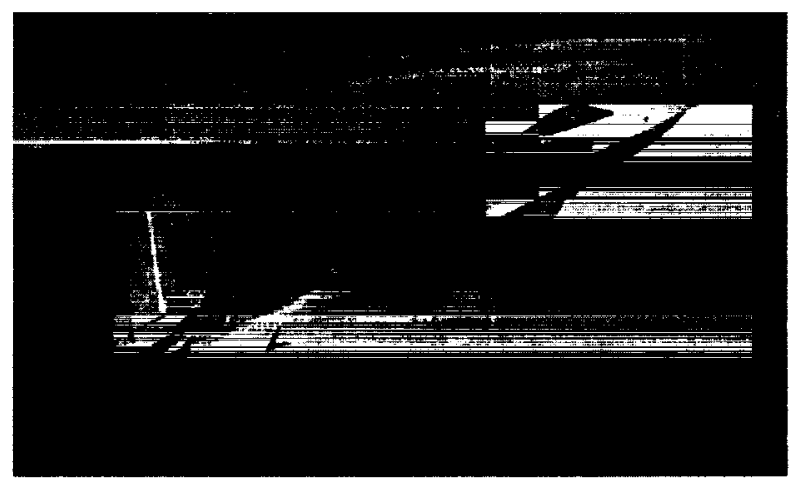

Figure 3. F-22 In Flight

Since very little data was available for the F-22 configuration (Figure 3), Ashley et. al. " ${ }^{11}$ estimated the buffeting of the F-22 fin by scaling F/A-18 pressure data. However, the shape of the F-22 engine inlet and wing leading edge differ from that of the F/A-18. Therefore, some assumptions were made in using the F/A-18 data for analyzing F-22 fin buffeting. Thus, the purpose of this paper is twofold: 1) to present some buffet and buffeting features of the F-22 configuration; and 2) provide comparisons between the F/A-18 and the F-22 fin buffet characteristics.
These characteristics are presented in various forms, such as root mean square (rms) values, power spectral density (PSD) cross-correlation and cross-spectral density (CSD) functions.

\section{Test Articles}

A 13.3\%-scale F-22 rigid model (Figure 4) was refurbished with a new starboard flexible tail and various types of instrumentation and mounted on a sting in the Transonic Dynamics Tunnel (TDT) at the NASA Langley Research Center. The model was tested at Mach numbers up to 0.12 and angles of attack between 16 and 42 degrees. Strouhal scaling was used in determining initial tunnel conditions.

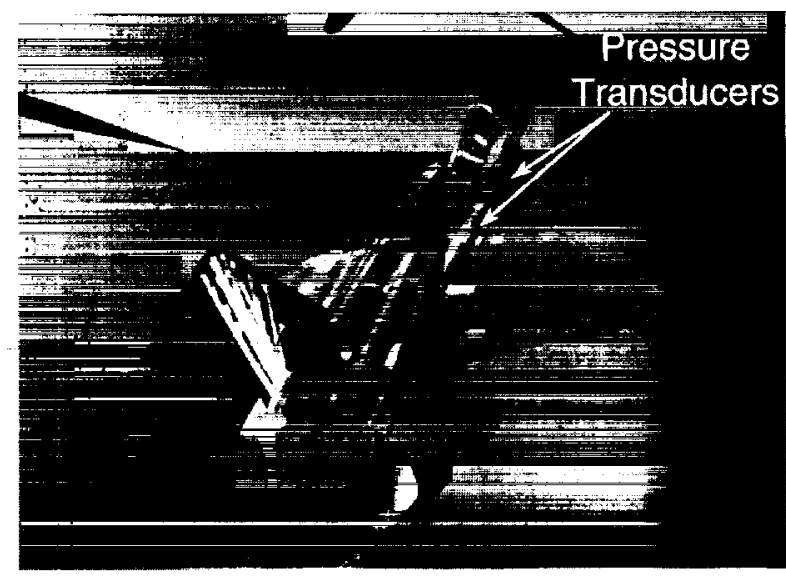

Figure 4. $13.3 \%$-Scale F-22 Model Mounted in the TDT

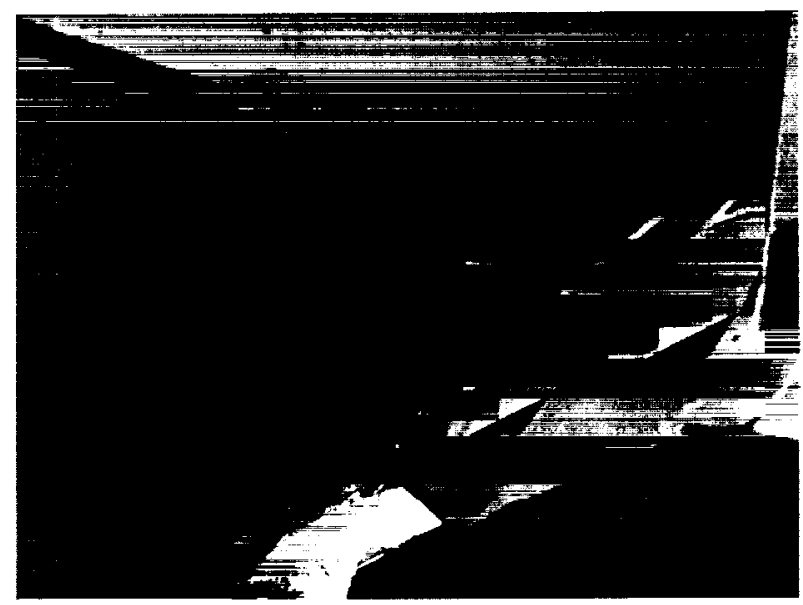

Figure 5. "Rigid" Port Fin With Pairs of Unsteady Pressure Transducers

The port fin (Figure 5), which was rigid relative to a dynamically-scaled flexible fin, was instrumented with 
pairs of surface-mounted unsteady pressure transducers arranged in a grid pattern (Figure 6). This pattern of stations was chosen for quantifying the unsteady buffet pressures that create motion of the fin in its first bending and first torsion modes.

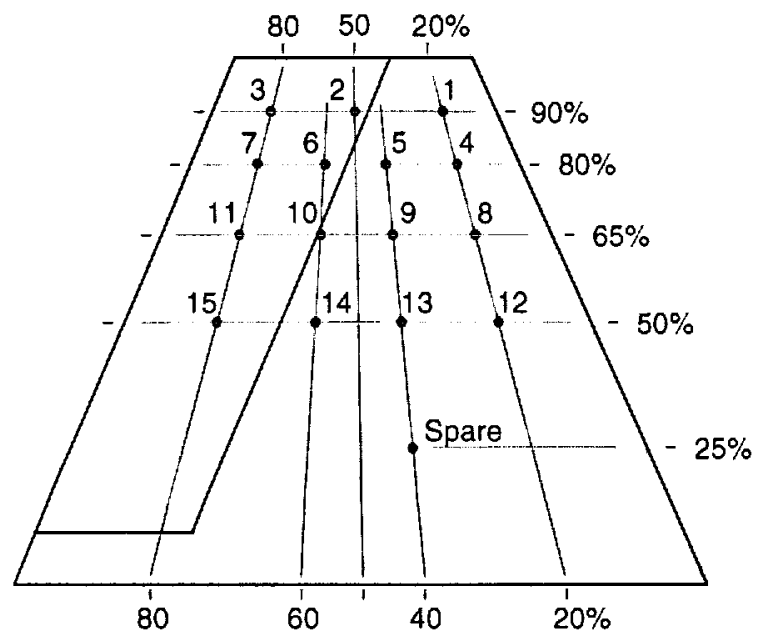

Figure 6. Stations of Pairs of Unsteady Pressure Transducers on the "Rigid" Port Fin

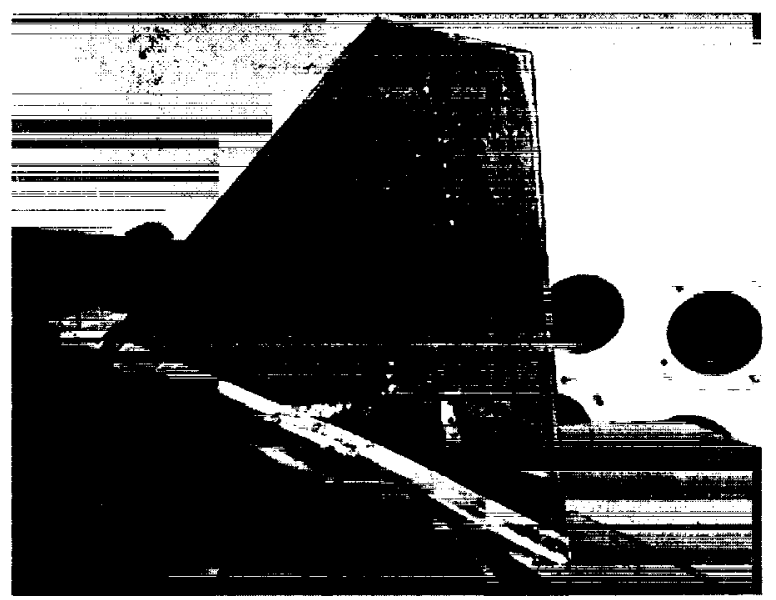

Figure 7. Flexible Starboard Fin With Active Rudder, Accelerometers, and Strain Gages

The starboard flexible fin (Figure 7) was scaled dynamically to an earlier F-22 configuration. The fin was fabricated using a honey-comb core construction with continuous skins. The rudder was actively driven by a hydraulic actuator. Accelerometers and strain gages were mounted on the fin (Figure 8) for measuring structural responses to the unsteady buffet pressures. A single unsteady pressure transducer was placed near the mid-span, mid-chord location on the inboard surface of the flexible fin for corroborating the pressures measured on the port fin and for assessing the downstream effects of the unsteady pressures measured at the root and LEX of the wing.

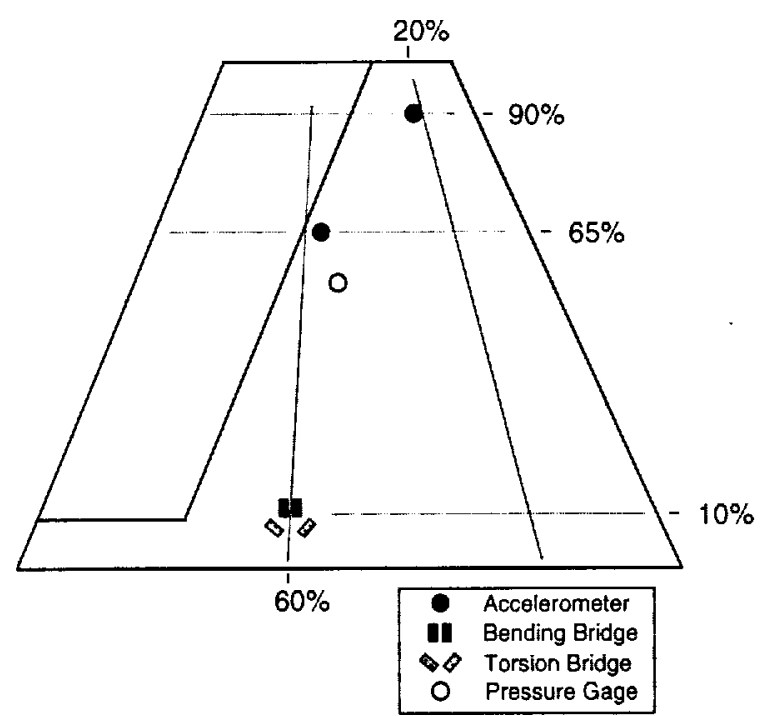

Figure 8. Arrangement of Accelerometers, Strain and Pressure Gages on Starboard Flexible Fin

\section{$\underline{\text { Buffet Features }}$}

Smoke was injected into the flow ahead of the model for visualizing the vortex pattern along the leading edge of the wing and engine inlets. The trajectory of the vortices observed over the F-22 are similar to the trajectories shown in Figure 2 for the F-15.

To illustrate fin buffet, power spectral density functions were computed of the surface unsteady pressures and the differential unsteady pressures at stations (Figure 6) on the "rigid" port fin. At the inboard transducer at Station 5 , an aerodynamic mode around $30 \mathrm{~Hz}$ begins forming at 22 degrees angle of attack (Figure 9). By 42 degrees angle of attack, this aerodynamic mode has grown in magnitude and shifted to a lower frequency value around $15 \mathrm{~Hz}$. Seen in Figure 10, the spectra of the buffet pressures on the outboard surface at Station 5 are similarly shaped; however, there are 2 noticeable differences: 1) the maximum pressure observed by the outboard transducers occurs at 22 degrees angle of attack (Figure 10) rather than at the higher angles of attack as observed by the inboard transducer (Figure 9); and 2) a second aerodynamic mode around $45 \mathrm{~Hz}$ is observed by the outboard transducers (Figure 10). Shown in Figure 11, the differential (inboard surface minus outboard surface) pressure at Station 5 is dominated by the pressure on the outboard surface (Figure 10) for angles of attack below 30 degrees. For higher angles of attack, the buffet pressure on the 
inboard surface (Figure 9) contributes more to the differential pressure (Figure 11). This feature indicates that the trajectory of the dominant vortex passes outboard of the fin at the lower angles of attack. As angle of attack is increased, this trajectory moves inboard eventually passing inboard of the fin.

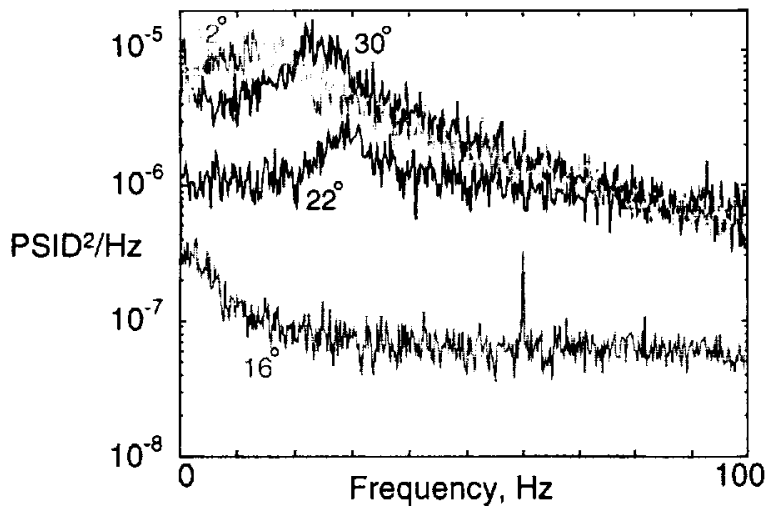

Figure 9. PSD of Buffet Pressures Measured at Station 5, Inboard Transducer, Mach 0.092

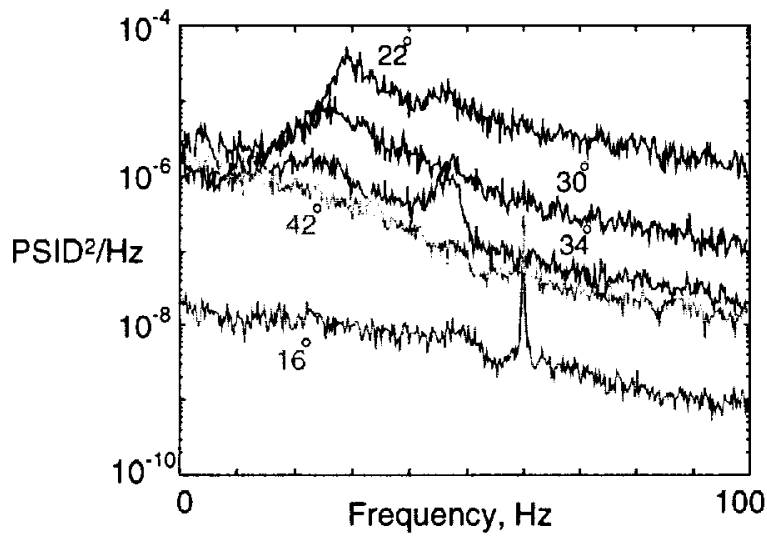

Figure 10. PSD of Buffet Pressures Measured at Station 5, Outboard Transducer, Mach 0.092

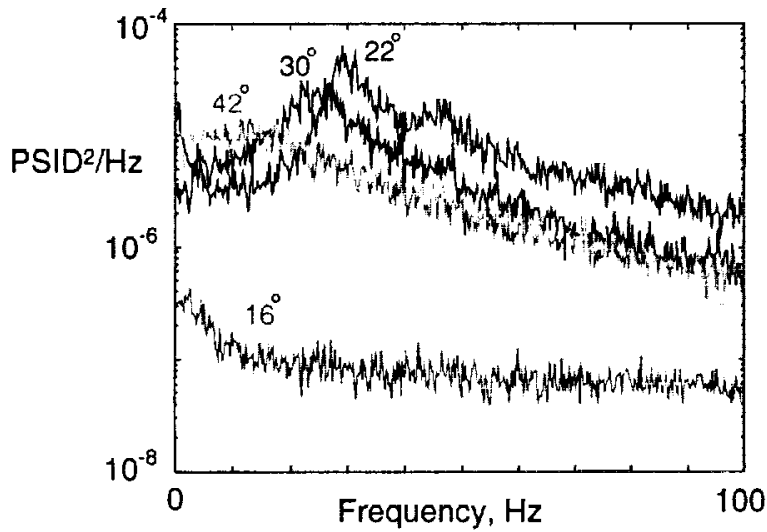

Figure 11. PSD of Differential Buffet Pressures Measured at Station 5, Mach 0.092

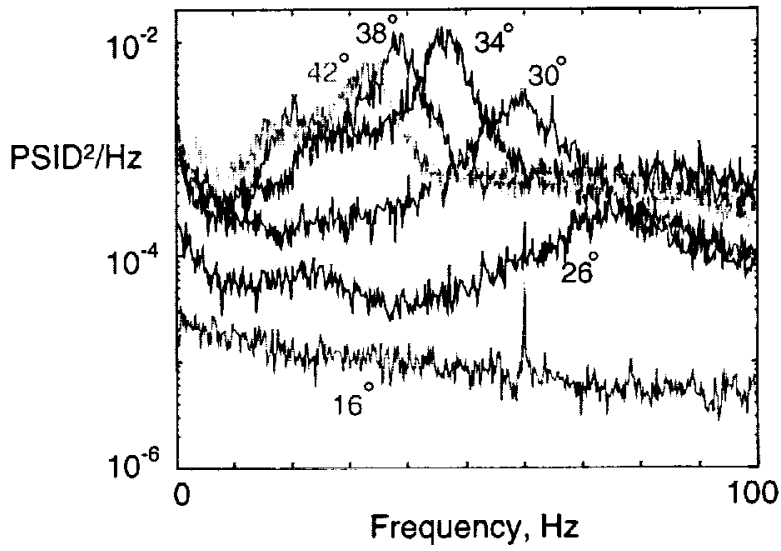

Figure 12. PSD of Buffet Pressures Measured at Wing 1/4-chord Root, Mach 0.092

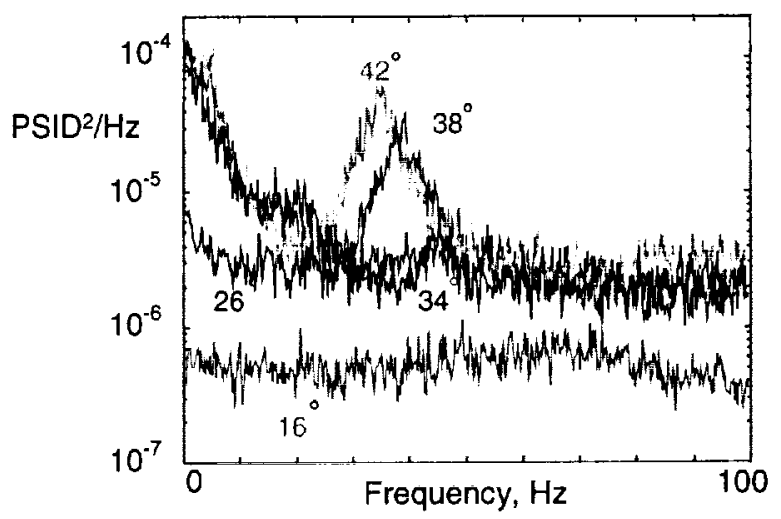

Figure 13. PSD of Buffet Pressures Measured on LEX Just Aft of Engine Inlet, Mach 0.092

In addition to measuring unsteady pressures on the fin, one unsteady pressure transducer was mounted on the LEX just aft of the engine inlet and another one mounted at the quarter-chord root of the starboard rigid wing (Figure 4). These transducers were placed in these positions for measuring unsteady pressures along the root and LEX of the wing. As shown in Figure 12, an aerodynamic mode at a frequency around $75 \mathrm{~Hz}$ begins forming above the starboard wing at 26 degrees angle of attack. As angle of attack is increased, this aerodynamic mode grows in magnitude while shifting to a lower frequency. This magnitude of this mode peaks around 34 degrees angle of attack.

In addition to this mode above the wing, an aerodynamic mode forms above the LEX just aft of the engine inlet, as shown in Figure 13. However, this mode forms at 34 degrees angle of attack, well after the mode above the wing was first measured. Also, the mode at the LEX increases in strength as angle of attack is increased above 38 degrees. However, the mode at the wing (Figure 12) decreases as angle of attack is 
increased above 38 degrees. Conceivably, these trends may indicate that the burst location initially lies between the two gages and approaches the engine inlet as angle of attack is increased. This concept is consistent with F/A-18 data. Another interesting feature is that the aerodynamic mode measured at the wing root (Figure 12) resides at a different frequency than the dominant aerodynamic mode measured at the fin (Figures 9 through 11). Although occurring at different frequencies, these two aerodynamic modes consistently illustrate a similar drop in magnitude and a shift to lower frequencies as angle of attack is increased (Figures 11 and 12).

For comparison, the rms values of the pressures measured at the wing root and fin are plotted versus angle of attack (Figure 14). While occurring at different angles of attack, the peak rms value of the pressure on the wing is more than one order of magnitude greater than the peak rms value of the buffet pressure at the fin.

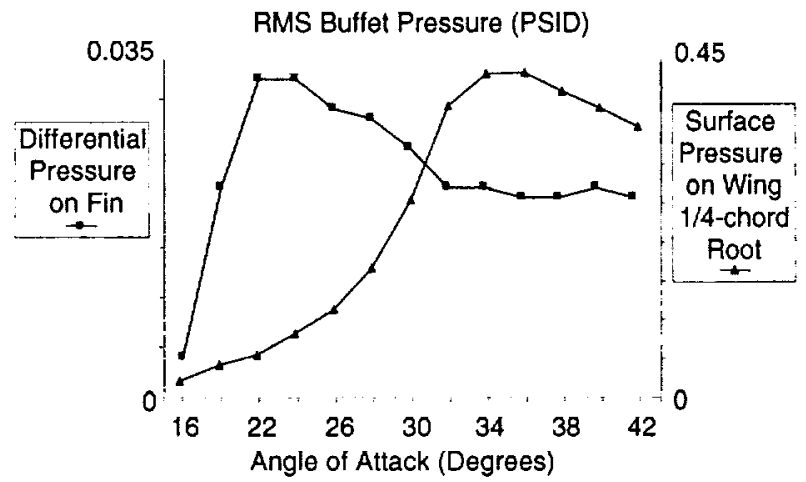

Figure 14. RMS Values of the Unsteady Buffet Pressures Measured at Station 5 (Differential) and at Wing 1/4-chord Root (Surface), Mach 0.092

As further evidence that significant changes occur between the buffet pressures at the fin and the unsteady pressures sensed at the wing root, the crosscorrelation function is computed (Figure 15). The maximum value of 0.2 indicates very little correlation between the pressures on the flexible fin and at the wing $1 / 4$-chord root location. Therefore, the vortex or vortices that create the primary buffet pressures at the fin (Figure 11) do not originate at the LEX. As observed during the test, the vortex originating near the leading edge of the wing (similar to Figure 2) engulfs the fin and is most probably the primary source of the fin buffet.

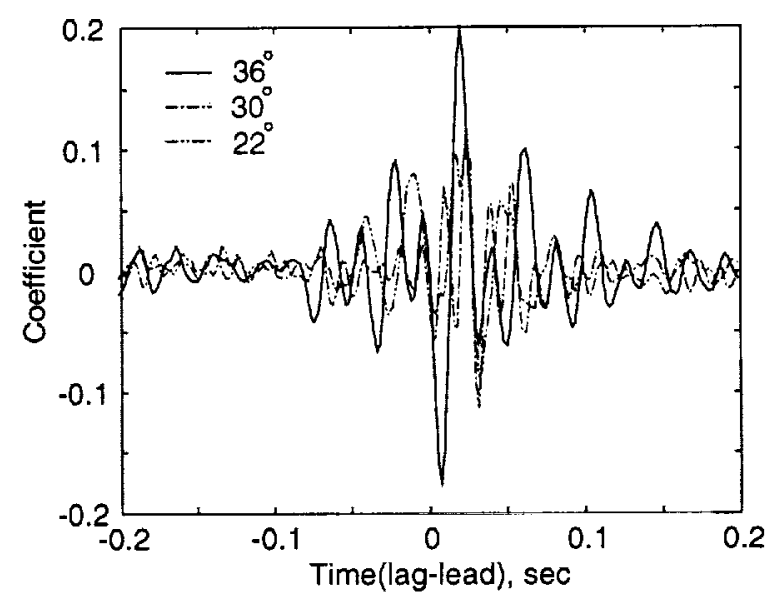

Figure 15. Cross-Correlation Function Between Unsteady Buffet Pressures Measured on Inboard Surface of Flexible Fin and Upper Surface of Wing at 1/4-chord Root, Mach 0.106

\section{Fin Buffeting}

The buffeting of the fin varied with angle of attack and occurred mainly in the first and second structural modes of the fin. The root bending moment (mode at $16 \mathrm{~Hz}$ in Figure 16) peaks at the higher angles of attack. Conversely, the torsion moment (mode around $30 \mathrm{~Hz}$ in Figure 17) peaks at the lower angles of attack.

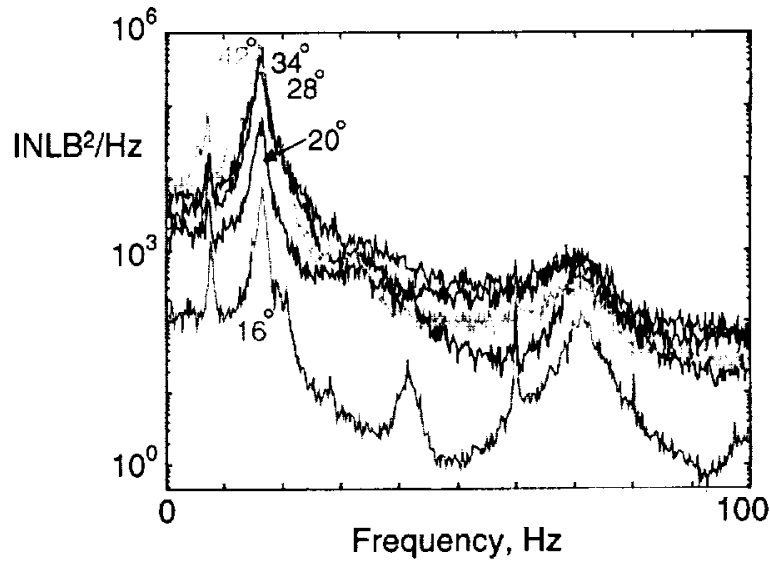

Figure 16. PSD of Root Bending Moment of Flexible Starboard Fin, Mach 0.092

The reason for these trends can be explained by examination of the buffet (forcing function) that is causing these modal responses. As illustrated previously in Figure 11, the aerodynamic forcing function on the fin varies with angle of attack. At 22 degrees, the aerodynamic mode at the fin (Figure 11) coincides with the frequency value of the torsion mode 
around $30 \mathrm{~Hz}$ (Figure 17). As angle of attack is increased, the aerodynamic mode (Figure 11) moves away from the torsion mode at $30 \mathrm{~Hz}$ and approaches the bending mode around $16 \mathrm{~Hz}$ (Figure 16). At 42 degrees angle of attack, the aerodynamic mode at the fin coincides with the first bending mode resulting in the highest measured value of root bending moment.

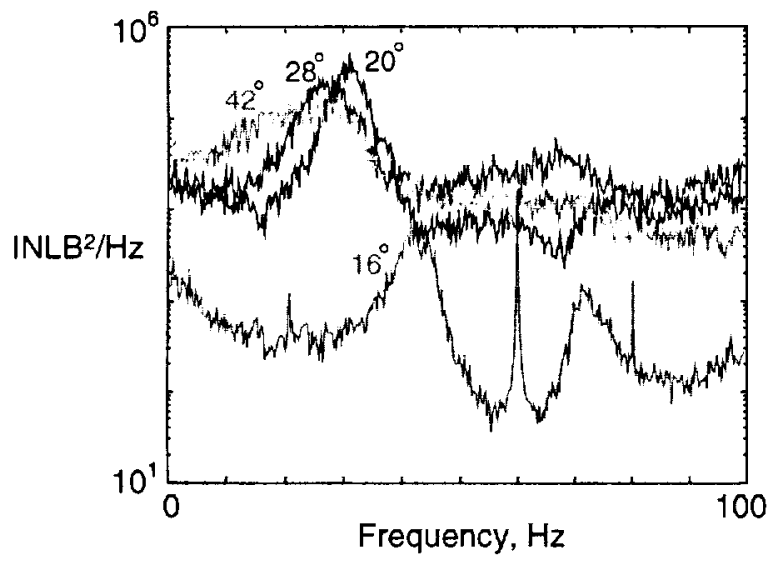

Figure 17. PSD of Root Torsion Moment of Flexible Starboard Fin, Mach 0.092

\section{Rudder Effectiveness at High Angles of Attack}

A series of tests was performed for assessing the force output and buffeting alleviation effectiveness of the rudder at high angles of attack. Initially, commands were sent to the rudder on the starboard fin while measuring the signals from response sensors located on the starboard fin (Figure 8). System identification techniques were employed for computing the frequency response functions between each sensor and the rudder command and deflection. Subsequently, these frequency response functions were used in designing control laws for alleviating fin buffeting.

Five different single-input single-output (SISO) control laws were tested. The phase and filtering were varied among these five control laws for verifying expected performances with actual alleviation results. Because of limited bandwidth of the rudder, alleviation of the first bending mode only was attempted. As shown in Figures 18 and 19, the root bending moment around $16 \mathrm{~Hz}$ was alleviated. As summarized in Figure 20, reductions in the rms value above $20 \%$ were achieved while using well below 1 degree (rms) of rudder deflection. These reductions indicate that the rudder is effective at all angles of attack tested between 24 and 38 degrees.

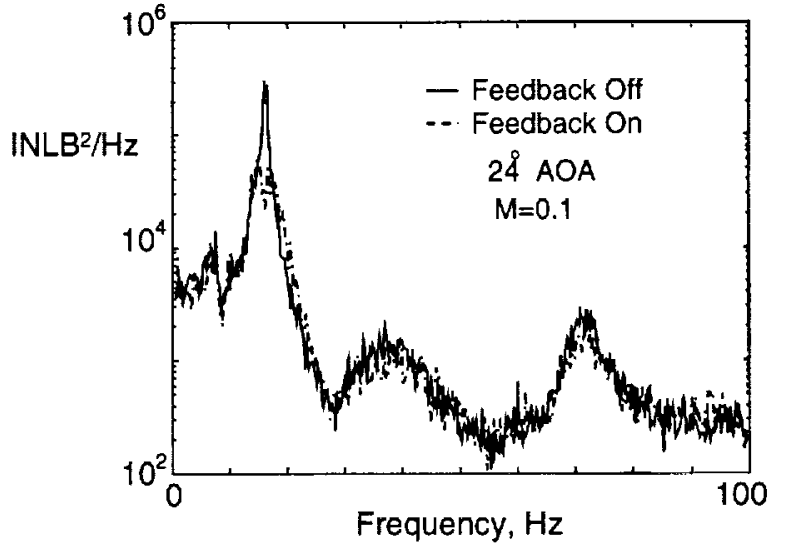

Figure 18. PSD of Root Bending Moment, Starboard Flexible Fin, Feedback Off and On Conditions, Mach 0.10 .

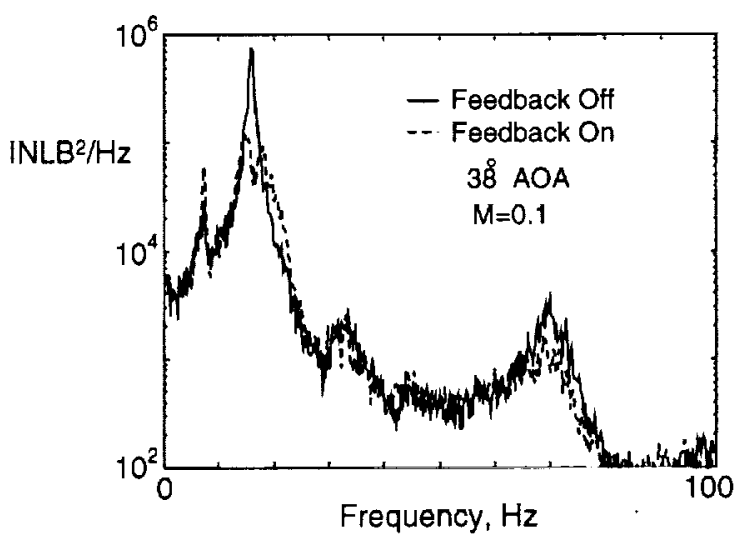

Figure 19. PSD of Root Bending Moment, Starboard Flexible Fin, Feedback Off and On Conditions, Mach 0.10.

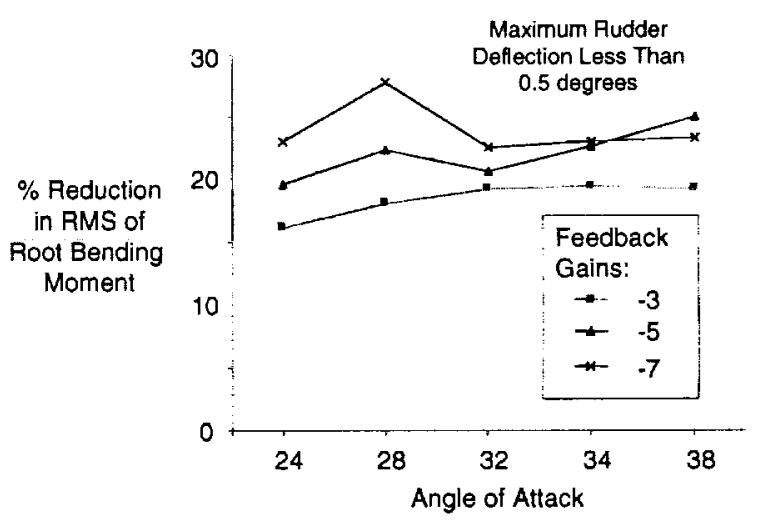

Figure 20. Reduction in Root Bending Moment, Starboard Flexible Fin, Feedback On Conditions Using Several Feedback Gains, Mach 0.10. 


\section{Spatial Features of the F-22 Fin Buffet Pressures}

In References 8-10 for an F/A-18 configuration, it was shown that the buffet pressures have the features of waves that travel along the fin. To study this feature in the present test, cross-spectral density (CSD) functions were computed for the differential buffet pressures at several stations for several angles of attack. A CSD consists of magnitude and phase plots and relates one signal to another. Thus, by examining the phase of the CSD function, one can determine how these two signals relate in terms of space (and time if the separation distance is known). For instance, if the phase is shown to be zero, then the identical features of these two signals are occurring at the same time although they are in different locations. If the phase is non-zero, then the identical features measured at the two locations occur at different times. The latter is a feature of a travelling wave.

\section{CSD: Differential Pressures @ Station 5 wrt Station 4}

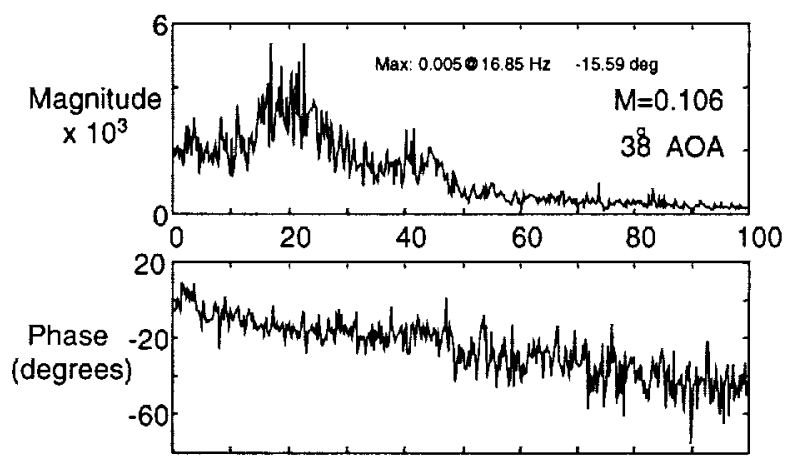

Frequency, $\mathrm{Hz}$

Figure 21. CSD, Differential Pressures, Station 5 with Respect to Station 4, Mach 0.106, 38 Degrees AOA

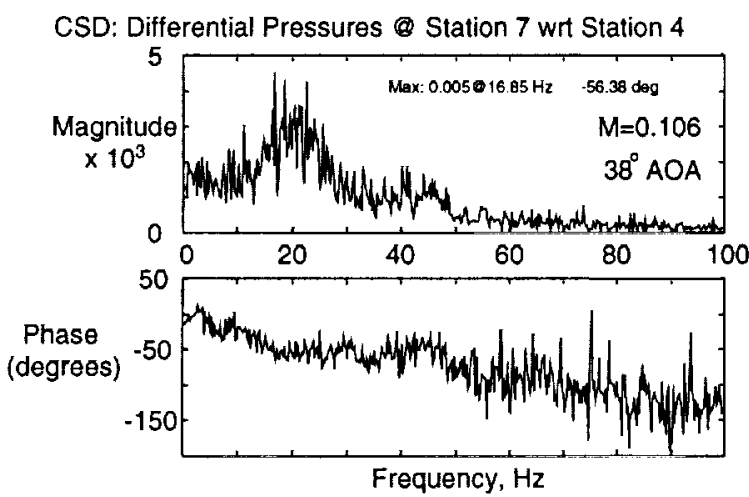

Figure 22. CSD, Differential Pressures, Station 7 with Respect to Station 4, Mach 0.106, 38 Degrees AOA
The wave phenomenon may be checked by determining whether the phase at a given frequency value triples when the distance between the two stations is tripled. As shown in Figure 21, the peak magnitude occurs in the vicinity of $20 \mathrm{~Hz}$ while the associated phase is around negative 20 degrees. When the separation distance is tripled, as is the case for the two stations whose CSD is shown in Figure 22, the phase at $20 \mathrm{~Hz}$ is around negative 60 degrees. Similar features were observed for the same stations while at a lower angle of attack.

Another metric for determining spatial "connectivity" is by using the coherence function. Although normalized to a maximum value of unity, the coherence function identifies the level of "connectivity" as a function of frequency. For instance, the coherence function between two separated stations that measured the same sinusoidal signal would appear as a spike (single vertical line) at the frequency of the sinusoid with a peak value of unity.

Seen by comparing Figures 23 and 24, the maximum value of coherence at 38 degrees angle of attack (Figure 23 ) is lower than the maximum value of coherence at 32 degrees angle of attack (Figure 24). This feature usually indicates that the vortices have lost energy as the model was moved from 32 degrees to 38 degrees angle of attack. It may also indicate that the burst location is closer to the nose when the model is at the higher angle of attack.

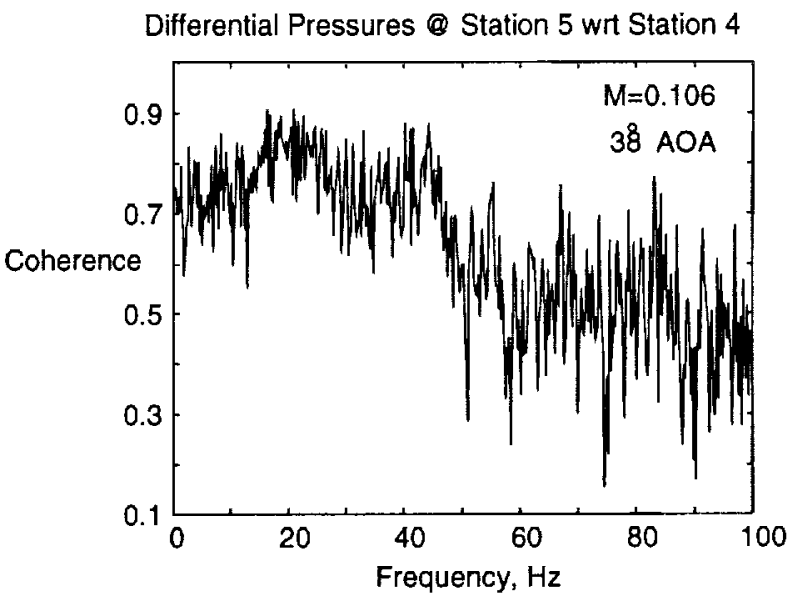

Figure 23. Coherence Function, Differential Pressure, Station 5 with Respect to Station 4, Mach 0.106, 38 Degrees Angle of Attack

Another difference apparent in the coherence functions (Figures 23 and 24) is that the peak between 20 and 30 $\mathrm{Hz}$ is broader at 38 degrees than at 32 degrees angle of 
attack. At 38 degrees angle of attack, there appears to be an aerodynamic mode contributing to the broadness of the peak (Figure 23).

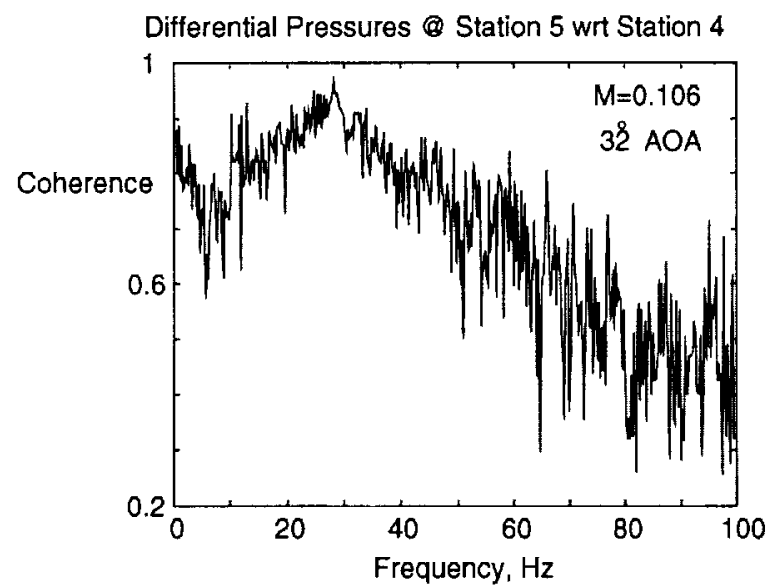

Figure 24. Coherence Function, Differential Pressure, Station 5 with Respect to Station 4, Mach 0.106, 32 Degrees Angle of Attack.

\section{Effects of Leading Edge Flap and Horizontal Tail Deflections}

In practice, leading edge flaps are deflected downward on the F/A-18 during high angle of attack maneuvers. Therefore, the effect of leading edge flap deflection on the F-22 unsteady fin pressures was investigated. In addition, the horizontal tail deflection angle was adjusted for assessing further impact on fin buffet.

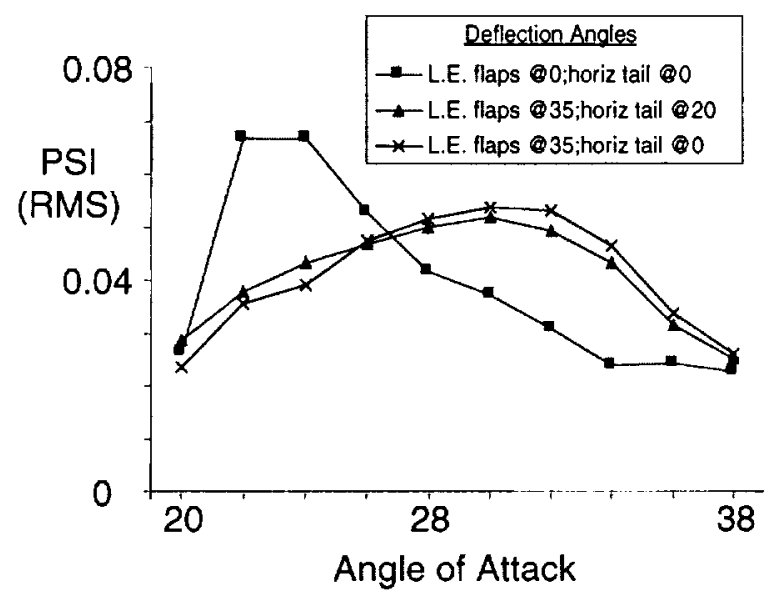

Figure 25. RMS Values of Buffet Pressures Measured at Station 1 (Differential), Mach 0.106, for 3 Cases of Leading Edge Flaps and Horizontal Tail Deflections

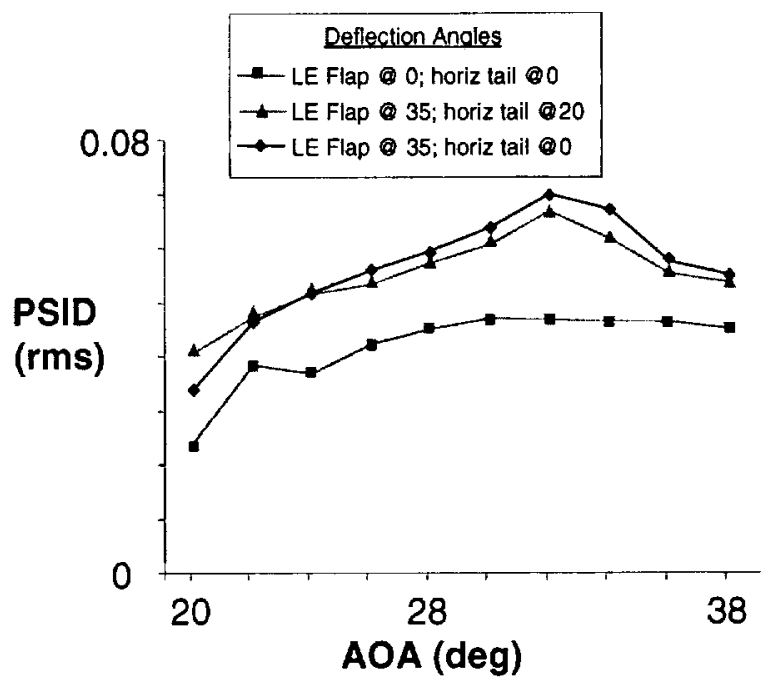

Figure 26. RMS Values of Buffet Pressures Measured at Station 12 (Differential), Mach 0.106, for 3 Cases of Leading Edge Flaps and Horizontal Tail Deflections

As shown in Figures 25 and 26, the deflection of the leading edge flap downward significantly impacts the fin buffet pressures. Near the tip of the fin (Figure 25), not only are the highest rms values of pressure shifted to the higher angles of attack, but the maximum pressure that occurs during the maneuver is greatly decreased. However, near mid-span of the fin, deflecting the flap actually increased the rms values of the buffet pressures (Figure 26). Deflecting the horizontal tail had little effect on the fin buffet (Figures 25 and 26).

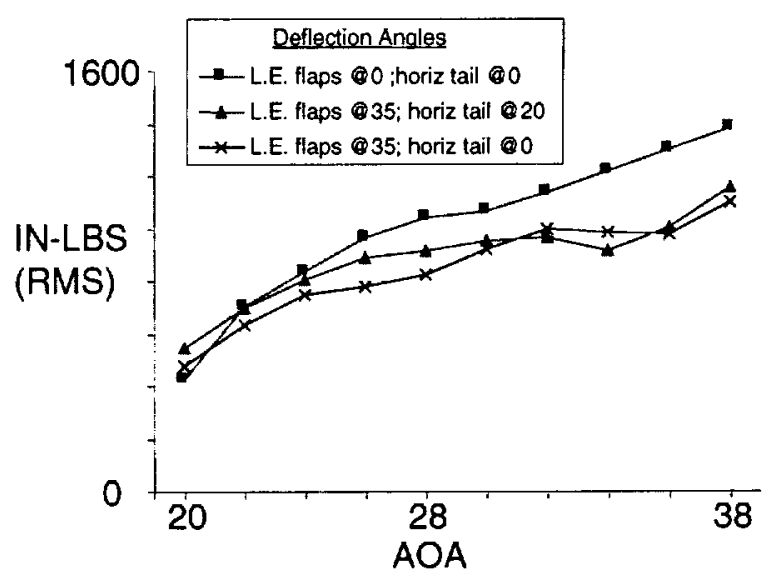

Figure 27. Root Bending Moment, Mach 0.106, for 3 Cases of Leading Edge Flaps and Horizontal Tail Deflections 
In terms of the structural response, similar effects were observed. Deflecting the leading edge flap reduced the root bending moment (Figure 27). Deflecting the horizontal tail had only minimal effect on the root bending moment of the fin (Figure 27).

\section{Comparisons With F/A-18 Data}

In examining an assumption made in Reference 11 , comparisons were made between the fin buffet pressures on the F/A-18 and F-22. For the F/A-18 fin (Figure 28), CSD functions (Figure 29) illustrate the traveling wave that was observed for the F/A-18. As seen in the CSD functions (Figures 21 and 22) for similarly located stations on the F-22 fin, the phase varies with distance between stations. However, there is one noticeable difference in the magnitude curves between the two aircraft configuration. Namely, that a second aerodynamic mode around $45 \mathrm{~Hz}$ seen in the PSDs (Figures 10 and 11) and the CSDs (Figures 21 and 22) for the F-22 is not present in the CSD (Figure 29) or PSDs (i.e., curve for 34 degrees in Figure 30) for the F/A-18.

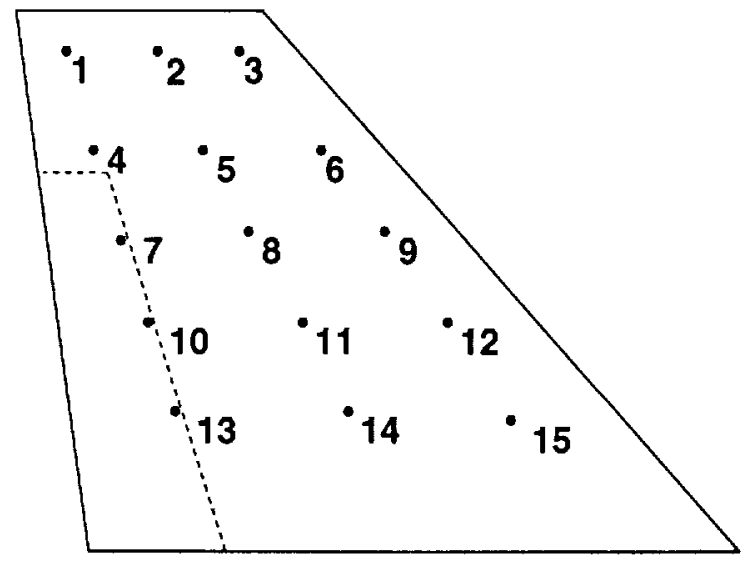

Figure 28. Pressure Transducer Stations, Port Rigid Tail, 1/6-Scale F/A-18 Model

This difference is noted also by comparison of the coherence functions for the F-22 and F/A-18 models. For the F/A-18, the peak magnitude of the coherence function (Figure 31) agrees with the maximum peak value for the F-22 (Figures 23 and 24). However, the width of this peak (Figure 31) for the F/A-18 is more narrow than the peak (Figures 23 and 24) for the F-22. Therefore, the spatial "connectivity" between adjacent stations on the F-22 appears "stronger" than for the F/A-18. Thus, the presence of a second vortex (around $45 \mathrm{~Hz}$ ) appears to influence the shape of the CSD and coherence functions of the fin buffet on the F22.

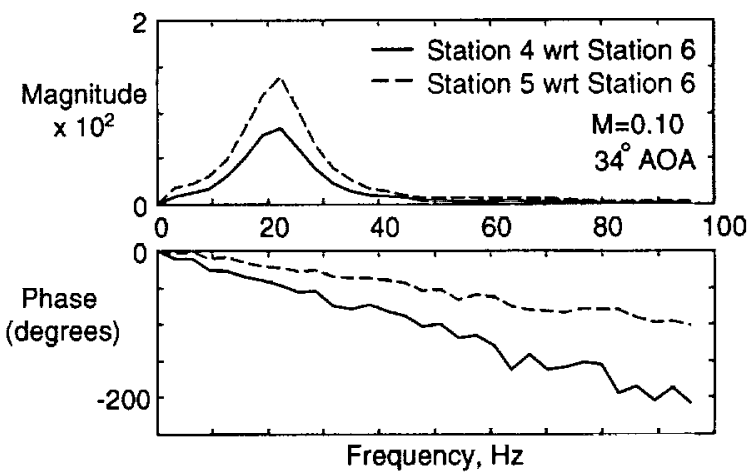

Figure 29. Cross-Spectral Density Functions, Stations on the Port Rigid Tail, 1/6-Scale F/A-18 Model, Mach $0.1,34$ Degrees AOA

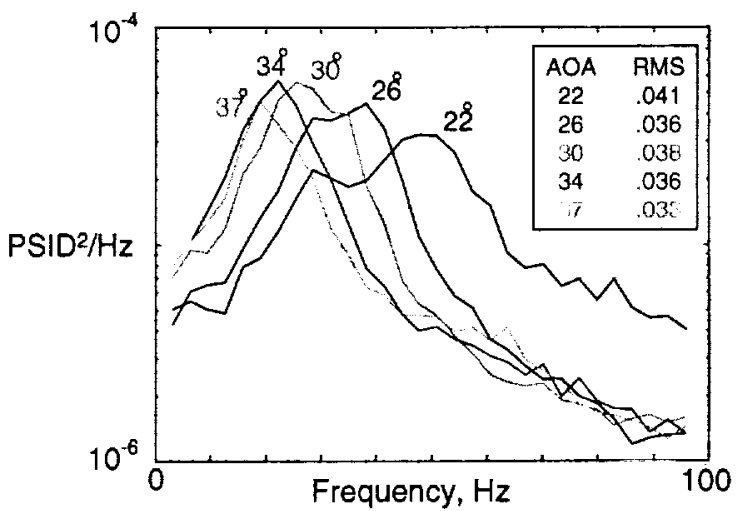

Figure 30. PSD of the Differential Buffet Pressures Measured at Station 6, F/A-18 Model, Mach 0.1

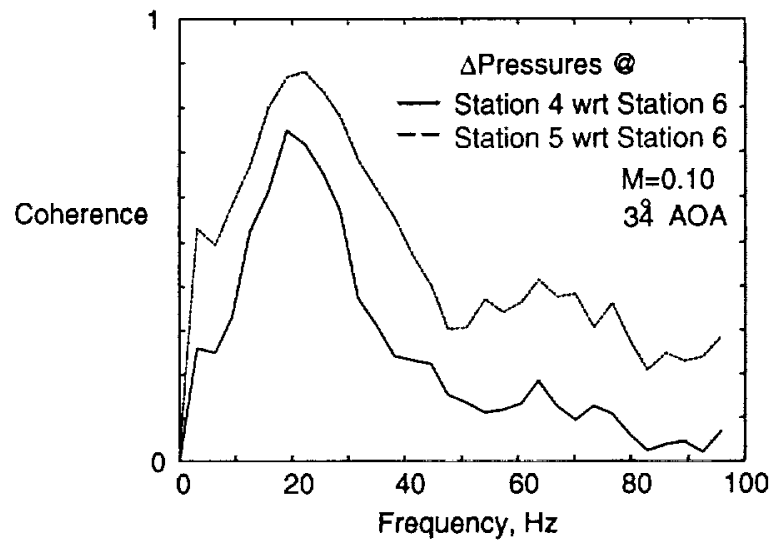

Figure 31. Coherence Functions, Stations on the Port Rigid Tail, 1/6-Scale F/A-18 Model, Mach 0.1, 34

Degrees AOA 


\section{Conclusions}

Based on test results of a $13.3 \%$-scale full-span F-22 model in the Transonic Dynamics Tunnel, some fin buffet and buffeting occur on the F-22 configuration. The flow ahead of the model was seeded with smoke for visualizing the trajectories of the vortices that form above the model at high angles of attack. Power spectral density, cross-spectral density, crosscorrelation, and coherence functions were computed of some fin pressures for presenting the fin buffet characteristics of the F-22 model. Power spectral density functions were computed of the root bending moment and root torsion moment of the starboard fin to illustrate the fin buffeting occurring at high angles of attack. Using feedback from an accelerometer on the starboard fin, the rudder was effective in reducing fin buffeting in the first bending mode. Furthermore, similarities and differences between fin buffet features of the F-22 and F/A-18 configurations were highlighted, illustrating the effects of multiple vortices on fin buffet features of the F-22.

\section{Acknowledgments}

The authors wish to express gratitude to the NASA Langley Research Center, the Air Force Research Laboratory, and the F-22 System Program Office for support of this wind-tunnel investigation.

\section{$\underline{\text { References }}$}

1 Triplett, W. E., "Pressure Measurements on Twin Vertical Tails in Buffeting Flow," AFWAL-TR82-3015, Vols I \& II, prepared for USAF Flight Dynamics Laboratory, April 1982.

2 Triplett, W. E., "Pressure Measurements on Twin Vertical Tails in Buffeting Flow," J. Aircraft, Vol. 20, No. 11, November 1983, pp. 920-925.

3 Zimmerman, N. H., and Ferman, M. A., "Prediction of Tail Buffet Loads for Design Application," Vols. I and II, Rept. No. NADC88043-60, July 1987.

4 Lee, B. H. K., Brown, D., Zgela, M., and Poirel, D., "Wind Tunnel Investigation and Flight Tests of Tail Buffet on the CF-18 Aircraft", AGARDCP-483, Advisory Group for Aerospace Research and Development Specialist's Meeting, Sorrento, Italy, April 1990.

5 Shah, G. H., "Wind-Tunnel Investigation of Aerodynamic and Tail Buffet Characteristics of Leading-Edge Extension Modifications to the
F/A-18," AIAA Atmospheric Flight Mechanics Conference, AIAA 91-2889, New Orleans, LA, August 12-14, 1991.

6 Pettit, C. L., Banford, M., Brown, D., and Pendleton, E., "Full-Scale Wind-Tunnel Pressure Measurements on an F/A-18 Tail During Buffet," Journal of Aircraft, Vol. 33, No. 6, NovemberDecember 1996, pp. 1148-1156.

7 Meyn, L. A. and James, K. D., "Full-Scale WindTunnel Studies of F/A-18 Tail Buffet," Journal of Aircraft, Vol. 33, No. 3, May-June 1996, pp. 589595.

8 Moses, R. W. and Pendleton, E., "A Comparison of Pressure Measurements Between a Full-Scale and a 1/6-Scale F/A-18 Twin Tail During Buffet," $83^{\text {rd }}$ Structures and Materials Panel Meeting, AGARDR-815, Loads and Requirements for Military Aircraft, 2-6 September 1996, Florence, Italy.

9 Moses, R.W. and Ashley, H., "Spatial Characteristics of the Unsteady Differential Pressures on $16 \%$ F/A-18 Vertical Tails," AIAA98-0519, 36 ${ }^{\text {th }}$ AIAA Aerospace Sciences Meeting and Exhibit, Reno, Nevada, January 12-15, 1998.

10 Moses, R. W. and Shah, G. H., "Spatial Characteristics of F/A-18 Vertical Tail Buffet Pressures Measured in Flight," AIAA-98-1956, $39^{\text {th }}$ AIAA/ASME/ASCE/AHS/ASC Structures, Structural Dynamics, and Materials Conference and Exhibit, Long Beach, CA, April 20-23, 1998.

11 Ashley, H., Rock, S. M., Digumarthi, R., and Chaney, K., "Further Study of Active Control for Fin Buffet Alleviation, With Application to F-22," WL-TR-97-3073, July 1997. 